\title{
Laser Therapy and Dementia: A Database Analysis and Future Aspects on LED-Based Systems
}

\author{
Daniela Litscher and Gerhard Litscher \\ Research Unit for Complementary and Integrative Laser Medicine, Research Unit of Biomedical Engineering in Anesthesia \\ and Intensive Care Medicine, and TCM Research Center Graz, Medical University of Graz, Auenbruggerplatz 29, \\ 8036 Graz, Austria \\ Correspondence should be addressed to Gerhard Litscher; gerhard.litscher@medunigraz.at
}

Received 26 March 2014; Accepted 9 April 2014; Published 29 April 2014

Academic Editor: Lu Wang

Copyright (C) 2014 D. Litscher and G. Litscher. This is an open access article distributed under the Creative Commons Attribution License, which permits unrestricted use, distribution, and reproduction in any medium, provided the original work is properly cited.

\begin{abstract}
Mainly because of the movement in the age pyramid, one can assume that the incidence of Alzheimer's disease or dementia in general will increase in the coming decades. This paper employs a database analysis to examine the profile of publication activity related to this topic. Two databases were searched: PubMed and Cochrane Library. About 600 papers related to the research area "dementia and laser" and about 450 papers related to the search terms "Alzheimer and laser" were found in these two most commonly used databases. Ten plus one papers are described in detail and are discussed in the context of the laser research performed at the Medical University of Graz. First results concerning the measurement of the transmission factor (TF) through the human skull of a new LED- (light emitting diode-) based system are presented (TF $=0.0434 \pm 0.0104(\mathrm{SD})$ ). The measurements show that this LED system (using the QIT (quantum optical induced transparency) effect) might be used in the treatment of dementia.
\end{abstract}

\section{Introduction}

Dementia is a widespread disease. According to data from 2013 there are 44.4 million people suffering from dementia worldwide. Mainly because of the movement in the age pyramid, one can assume that the incidence of dementia will increase in the coming decades. Experts mentioned that in 2030 an estimated 75.6 million and, in 2050, 135.5 million people will suffer from dementia. The biggest problem is the increase in the developing countries. There are already $62 \%$ of people with dementia living in developing countries. The fastest growth in the elderly population is taking place in China, India, and their south Asian and Western Pacific neighbors. Experts warn that the whole percentage of people with dementia will increase to $71 \%$ in 2050 in those countries [1].

In Europe, for example, the Austrian society for Alzheimer's disease states that this particular disease is responsible for $60-80 \%$ of dementia cases. In Austria, currently 1 billion Euros is necessary for the therapy of dementia patients every year [2]. In Switzerland dementia is also a huge financial problem. On the homepage of the Swiss Alzheimer Union it is mentioned that every year about 7 billion Swiss francs is needed for the therapy of dementia patients [3]. Statistics from Germany (German Federal Ministry of Family Affairs, Senior Citizen, Women and Youth) show that one in three persons aged over 90 suffers from dementia. Experts warn that by 2050 the number of people in Germany suffering from dementia will increase to 3 million [4].

In the United States of America Alzheimer's disease (type of dementia) is the 6th leading cause of death. Total payments for health care, long-term care, and hospice for people with Alzheimer's and other types of dementia are projected to increase from $\$ 203$ billion in 2013 to $\$ 1.2$ trillion in 2050 (in current dollars) [5].

The goal of the present study was to give a short scientific overview on the topic laser therapy and dementia and to take new LED- (light emitting diodes-) based systems which use the quantum optical induced transparency effect into account. Therefore a database analysis in scientific databases 
was performed and the transmission factor (TF) of a new LED-based system through the human skull was calculated.

\section{Current Scientific Research}

A search of two scientific databases PubMed and Cochrane Library, on February 19, 2014, yielded more than 600 publications related to the topic laser and dementia. The topic laser and Alzheimer listed more than 450 scientific publications. The other search terms were "Alzheimer (or dementia) and laser; laser therapy; laser therapy transcranial; laser acupuncture; red laser; yellow laser" (see Figures 1 and 2).

Ten referenced publications (PubMed) with the terms "red laser" or "yellow laser" and "dementia" [6-15] are described in detail and cited in the list of references. Compared to laser or laser therapy in general this number of publications is very small. In the following, the 10 publications from the two databases will be discussed. At first 7 publications found with the search terms "dementia" and "red laser" are described.

Most of these studies deal with different laser methods for diagnostic purposes but are not directly related to laser therapy.

In the first paper, the authors state that occlusive brain ischemia and microstrokes are a major cause of dementia in older patients. In this in vivo animal experimental study twophoton laser-scanning microscopy and transgenic mouse models expressing cell type specific reporters were used to examine ischemia-related insults [6]. In the study by Sommer et al. [7] the authors wanted to show a potential relevance of their in vitro experiments in the treatment of Alzheimer's disease (AD). Amyloid-beta $(\mathrm{A} \beta)$ was internalized into human neuroblastoma (SH-EP) cells which were then irradiated with moderately intense $670 \mathrm{~nm}$ laser light $\left(1000 \mathrm{~W} / \mathrm{m}^{2}\right)$ and/or treated with epigallocatechin gallate (EGCG). In the irradiated cells, the authors found that $\mathrm{A} \beta(42)$ aggregate amounts were significantly lower than in nonirradiated cells. They concluded that irradiation with moderate levels of $670 \mathrm{~nm}$ light and EGCG supplementation complementarily reduces $\mathrm{A} \beta$ aggregates in SH-EP cells.

An important study was published in the renowned scientific journal Lancet in the year 2003 [8]. The authors of the paper entitled "Cytosolic beta-amyloid deposition and supranuclear cataracts in lenses from people with Alzheimer's disease" obtained postmortem specimens of eyes and brain from nine individuals with $\mathrm{AD}$ and eight controls without the disorder and samples of primary aqueous humour from three people without the disorder who were undergoing cataract surgery. Among others they used enhanced laser desorption ionisation. $A \beta 1-40$ and $A \beta 1-42$ were found in the lenses of all subjects, at concentrations comparable to those in the brain, and $A \beta 1-40$ was found in primary aqueous humour at concentrations comparable to that in cerebrospinal fluid. The authors concluded that since $\mathrm{A} \beta$ was found in the cytosol of lens fibre cells of people with Alzheimer's disease, lens A $\beta$ might promote regionally specific lens protein aggregation,

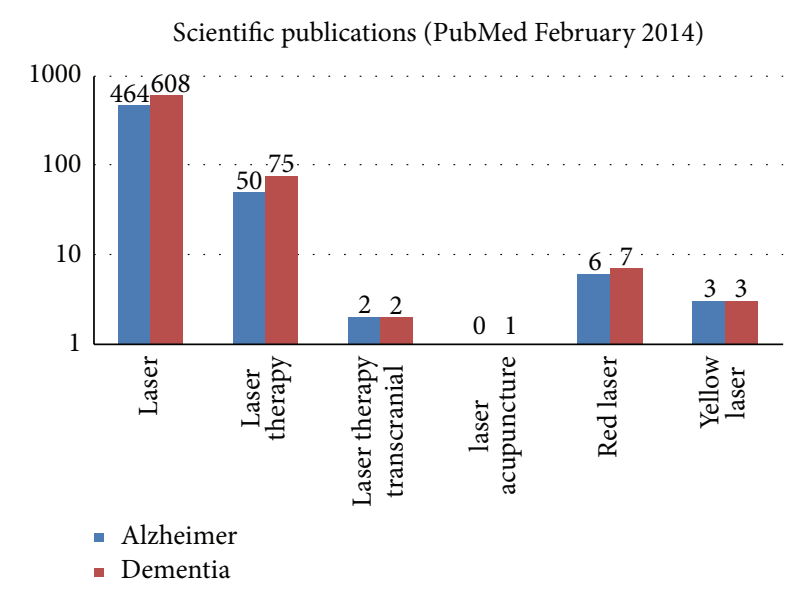

FIGURE 1: Scientific publications related to the topic in the scientific database PubMed.

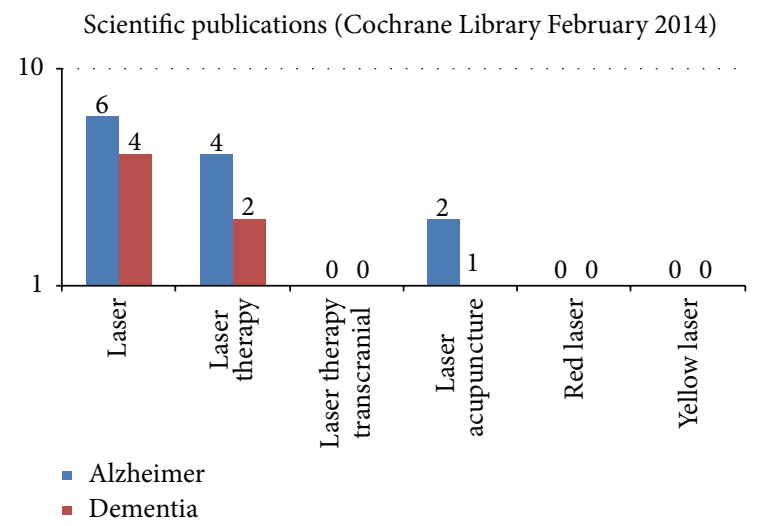

FIGURE 2: Scientific publications related to the topic in the scientific database Cochrane Library.

extracerebral amyloid formation, and supranuclear cataracts [8].

A paper by Miyakawa et al. from the year 2000 also deals with the role of blood vessels and the deposition of $\mathrm{A} \beta$ protein in producing pathological changes in the brain with $\mathrm{AD}$ [9]. Laser scanning microscopy revealed a close relationship between $\mathrm{A} \beta 40$ and the vascular network. The amount of mature plaques was reportedly correlated with the severity of dementia in Alzheimer's patients. Therefore, the authors concluded that accumulation of $\mathrm{A} \beta 40$ associated with blood vessels may play a critical role in the development of AD. Another paper entitled "Confocal observation of senile plaques in Alzheimer's disease: senile plaque morphology and relationship between senile plaques and astrocytes" was published by Kato et al. in 1998 [10]. Senile plaques in the brains with $\mathrm{AD}$ were examined by confocal laser scanning microscopy [10]. Chiu and Liu [11] measured the erythrocytes deformability using a laser visco diffractometer (Vidometer). They found that the deformability of glucose-6-phosphate dehydrogenase- (G6PD-) deficient erythrocytes was drastically reduced by hydroxyl radicals and hypothesized that as a consequence of enhanced susceptibility to oxidative stress, 
G6PD-deficient individuals have lower antioxidant levels, particularly vitamin $C$, than normal individuals. An older publication from 1995 deals with the topic "Mitochondrial constituents of corpora amylacea and autofluorescent astrocytic inclusions in senescent human brain" [12]. Immunofluorescence labeling and laser scanning confocal microscopy demonstrated consistent colocalization of the mitochondrial proteins, sulfite oxidase, and heat shock protein 60 , to both corpora amylacea (CA) and the autofluorescent astroglial inclusions. These observations suggest that Gomori-positive granules may be structural precursors of CA in the senescent human brain, and degenerated mitochondria within periventricular astrocytes give rise to autofluorescent cytoplasmic granules and CA [12].

In the following 3 papers found with the search terms "dementia" and "yellow laser" are mentioned.

The two-photon laser scanning microscopy is often used for chronic in vivo studies. In this study Scheibe et al. used an upright two-photon microscope complemented with a software-controlled stage-rotation instead of a conventional stage for chronic in vivo imaging in the brain of transgenic mouse models of AD [13]. This experimental setup allowed the authors to eliminate deviations in sample orientations between consecutive imaging sessions, thus facilitating comparisons between three-dimensional image stacks taken at different time points [13]. One article by Li et al. deals with the "Construction of recombinant plasmid harboring APP717 mutation and preliminary study of APP proteolysis" [14]. The authors investigated the pathogenesis of $\mathrm{AD}$ and studied the enzymatic progress of amyloid precursor protein (APP) using laser confocal microscopy. In summary, the recombinant plasmid could be a useful tool to further study the cleavage mechanism of APP and to explore the pathogenesis of AD. The last publication which is listed in the literature deals with the "Use of YFP to study amyloid-beta associated neurite alterations in live brain slices" [15]. Again, laser scanning confocal microscopy was used by the authors of this study. Their acute slice preparation model should prove to be a useful tool to explore the pathophysiology of $A \beta$-related axonal, dendritic, and synaptic dysfunction.

A very important, maybe the most important paper related to the topic laser and dementia was released in 2013. "Low-level laser therapy rescues dendrite atrophy via upregulating BDNF expression: implications for Alzheimer's disease" was published in the Journal of Neuroscience [16]. The authors stated that the so-called low-level laser therapy (LLLT) was demonstrated to regulate neuronal function both in vitro and in vivo. They found that LLLT rescued neurons loss and dendritic atrophy via upregulation of brain-derived neurotrophic factor (BDNF) in both $\mathrm{A} \beta$-treated hippocampal neurons and cultured APP/PS1 mouse hippocampal neurons. They also found that photoactivation of transcription factor CRE-binding protein (CREB) increased both BDNF mRNA and protein expression, since knockdown CREB blocked the effects of LLLT. Among other things these studies suggest that upregulation of BDNF with LLLT can ameliorate $A \beta$ induced neurons loss and dendritic atrophy, thus identifying a novel pathway by which LLLT protects against $\mathrm{A} \beta$-induced neurotoxicity [16].
In addition to the ten papers mentioned before, there is another very interesting article listed in the scientific database PubMed [17]. In the progression of $\mathrm{AD}$, oxidative stress and inflammation are important processes. The authors mentioned that recent studies have implicated the role of $\mathrm{A} \beta$ in mediating these processes. The objective of their study was to examine whether laser light with a wavelength of $632.8 \mathrm{~nm}$ was able to abrogate the oxidative and inflammatory responses induced by $\mathrm{A} \beta$. Primary rat astrocytes were exposed to helium-neon laser, followed by the treatment with oligomeric $\mathrm{A} \beta$. The author's data showed that, amongst others, laser light suppressed $\mathrm{A} \beta$-induced superoxide production.

Another paper from the year 2012 deals with low-power laser irradiation (LPLI). Apoptosis induced by $\mathrm{A} \beta$ is thought to associate with the pathogenesis of $\mathrm{AD}$. The mechanisms of LPLI to reduce $\mathrm{A} \beta$-induced apoptosis are still unclear. The authors reported a novel molecular mechanism by which LPLI attenuates $\mathrm{A} \beta(25-35)$-induced apoptosis. The data demonstrate that LPLI has a prosurvival effect on $\mathrm{A} \beta$-induced apoptosis and may be an effective therapeutic strategy in treating $\mathrm{AD}[18]$.

\section{Future Aspects-LED-Based Systems}

At the moment there is only one paper concerning light emitting diodes and dementia (Alzheimer) in the scientific databases PubMed and Cochrane Library [19]. However, this paper uses LED not for therapeutic reasons but for stimulation purposes. The authors investigated 12 patients with $\mathrm{AD}$ and their age and sex-matched controls. The goal of the study was to analyze whether Alzheimer's patients experienced a problem spontaneously planning an upcoming movement. The authors of the study state that the patients with $\mathrm{AD}$ revealed slower and less efficient movements than controls. To the best of our knowledge, up to now a LEDbased system has never been used in extensive investigations concerning dementia, and therefore no other parameters are available.

Within this study, a new LED-based system was investigated with regard to its transmission factor on the human skull for the first time. Similar to a previous study [20], the transmission through the skull was measured with commercially available methods (BL-10 L luxmeter, Volt Craft, Hirschau, Germany). The registrations on the highest area of the skull $(1.6 \times 6.5 \mathrm{~cm}$ around electroencephalogram position $C_{z}$ ) were reproducible, and in total nine measurements were performed using the new LED-based system medlouxx (laneg GmbH, Schönwalde, Germany) (see Figure 3). This system emits noncoherent, infrared LED radiation with a wavelength of $830 \mathrm{~nm}$. The output power of the LEDapplicator is $760 \mathrm{~mW}$, and the LED class of the system is $1 \mathrm{M}$ according to IEC 60825-1:A2. The absolute value of the measurement inaccuracy concerning the LED output power is $20 \%$.

The measurements were performed at a human skull provided by the Department of Anatomy, Medical University of Graz. 


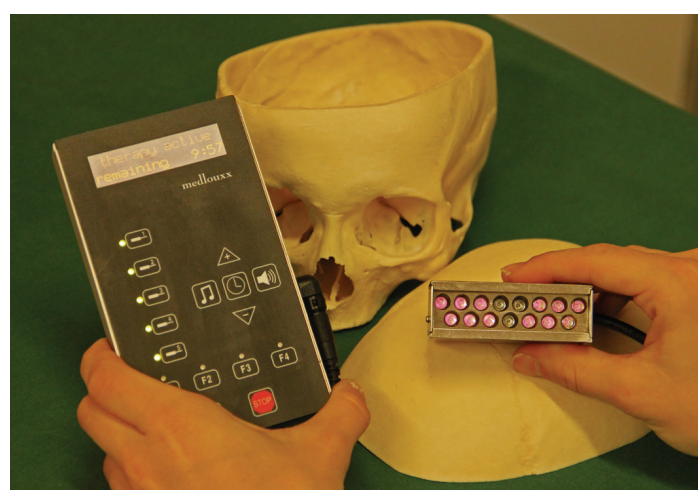

FIgURE 3: The medlouxx system including the LED applicator.

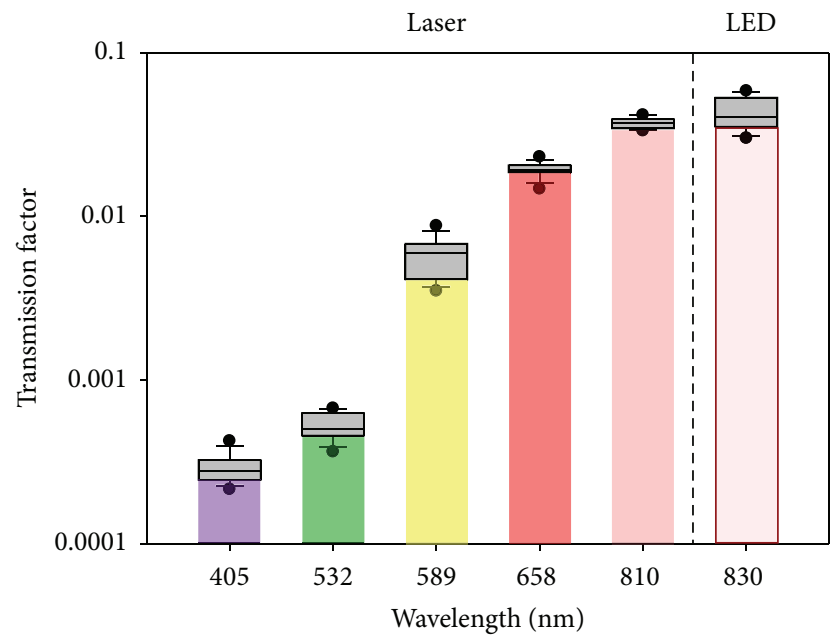

FIGURE 4: Transmission factor of different types of laser (modified from [20]) and of the new medlouxx system (LED). The results from violet $(405 \mathrm{~nm})$, green $(532 \mathrm{~nm})$, yellow $(589 \mathrm{~nm})$, red $(658 \mathrm{~nm})$, and infrared $(810 \mathrm{~nm})$ lasers are based on a previous study [20]. The system based on LED showed the highest mean value of the transmission factor $(830 \mathrm{~nm}$ infrared light). The lines in the boxes represent the median, the ends of the boxes define the 25th and 75th percentile, and the error bars define the 10th and 90th percentile.

Data were analyzed using SigmaPlot 12.0 software (Systat Software, Chicago, USA) and are presented as box plots. One way repeated measures ANOVA was used for statistical analysis, and Tukey's test was used for post hoc analysis. A $P$ value of $<0.05$ was considered significant.

The results of the measurements can be seen in Figures 4 and 5 .

It is interesting that the LED based system which uses a special physical effect, the so-called QIT- (quantum optical induced transparency-) effect [21-23], has the highest TF $(0.0434 \pm 0.0104(\mathrm{SD}))$. In the literature, it is described that this electromagnetic effect has become an important tool to control the optical properties of dense media and that it has the potential to enhance the transparency contrast by a factor of five [23]. Compared to our laser measurements, the new LED system has indeed a high TF. There is a significant difference between red laser light $(658 \mathrm{~nm}$;

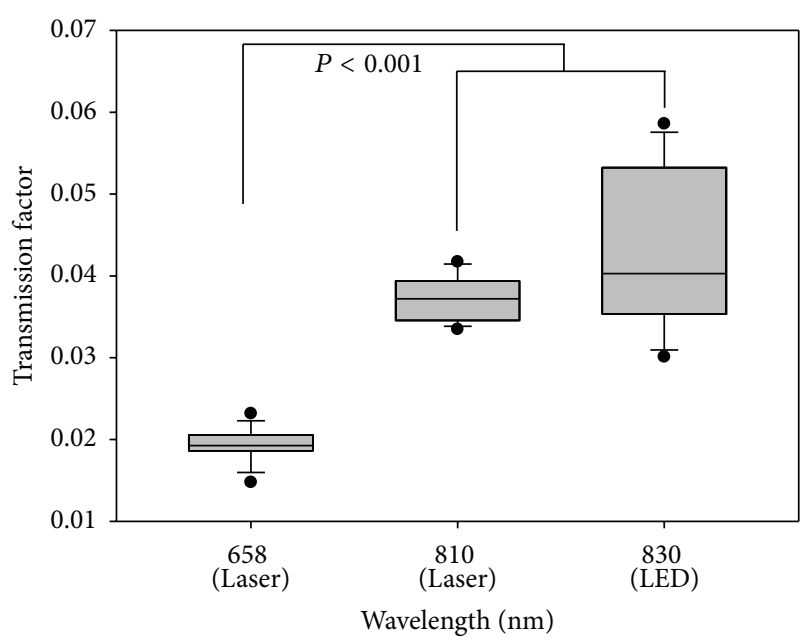

FIGURE 5: Transmission factor of red $(658 \mathrm{~nm})$ and $810 \mathrm{~nm}$ infrared laser light and of $830 \mathrm{~nm}$ infrared LED radiation. For further explanations, see Figure 4.

$\mathrm{TF}=0.0194 \pm 0.0023)$ and $830 \mathrm{~nm}$ infrared $\mathrm{LED}$ radiation (compare Figure 5). However, the TF of infrared laser light $(810 \mathrm{~nm} ; 0.0373 \pm 0.00299)$ and infrared LED radiation does not differ significantly.

\section{Conclusion}

At first it should be mentioned that there are many scientific publications on the topic dementia (resp., Alzheimer) and laser diagnostics and therapy. However, there is one very important study about low-level laser therapy and Alzheimer's disease in general [16]. The other described papers do not deal with laser irradiation as a treatment method but with the research on dementia with diagnostic laser methods [6-15]. We believe that the prerequisites (TF) for yellow, red, and infrared irradiation can be considered as given so that dementia research can be performed. In this context, also the new LED based system medlouxx fulfills the requirement "transmission factor." Further research on this topic is absolutely necessary.

\section{Conflict of Interests}

The authors declare that there is no conflict of interests regarding the publication of this paper.

\section{Acknowledgments}

The scientific investigations were supported by the German Academy of Acupuncture (President Professor Frank Bahr, Munich, Germany) and by the Austrian Federal Ministries of Science, Research and Economy and Health (project title Evidence-Based High-Tech Acupuncture and Integrative Laser Medicine for Prevention and Early Intervention of Chronic Diseases). In addition, this study is a forerunner of the project Transcranial Photomedicine in Neurorehabilitation supported by the Austrian Federal Ministry of 
Science, Research and Economy. The authors would like to thank Professor Georg Feigl, M.D., from the Department of Anatomy, and Ms. Ingrid Gaischek, M.S., both from the Medical University of Graz, for their valuable help.

\section{References}

[1] “Alzheimer's Disease International,” 2014, http://www.alz.co.uk/ research/statistics.

[2] "Österreichische Alzheimer Gesellschaft," 2014, http://www .alzheimer-gesellschaft.at/index.php?id=46.

[3] "Schweizerische Alzheimervereinigung," 2014, http://www .alz.ch/index.php/zahlen-zur-demenz.html.

[4] "Deutsches Bundesministerium für Familie, Senioren, Frauen und Jugend," 2014, http://www.wegweiser-demenz.de/meldungen-und-zahlen.html.

[5] "Alzheimer's Association," 2014, http://www.alz.org/alzheimers_diseasefacts_and_figures.asp.

[6] F. Kirchhoff, F. Debarbieux, C. Kronland-Martinet, G. R. Cojocaru, and A. Popa-Wagner, "Combined two-photon laserscanning microscopy and spectral microCT X-ray imaging to characterize the cellular signature and evolution of microstroke foci," Romanian Journal of Morphology and Embryology, vol. 53, supplement 3, pp. 671-675, 2012.

[7] A. P. Sommer, J. Bieschke, R. P. Friedrich et al., “670 nm laser light and EGCG complementarily reduce amyloid- $\beta$ aggregates in human neuroblastoma cells: basis for treatment of alzheimer's disease?" Photomedicine and Laser Surgery, vol. 30, no. 1, pp. 54-60, 2012.

[8] L. E. Goldstein, J. A. Muffat, R. A. Cherny et al., "Cytosolic $\beta$ amyloid deposition and supranuclear cataracts in lenses from people with Alzheimer's disease," The Lancet, vol. 361, no. 9365, pp. 1258-1265, 2003.

[9] T. Miyakawa, T. Kimura, S. Hirata et al., "Role of blood vessels in producing pathological changes in the brain with Alzheimer's disease," Annals of the New York Academy of Sciences, vol. 903, pp. 46-54, 2000.

[10] S. Kato, T. Gondo, Y. Hoshii, M. Takahashi, M. Yamada, and T. Ishihara, "Confocal observation of senile plaques in Alzheimer's disease: senile plaque morphology and relationship between senile plaques and astrocytes," Pathology International, vol. 48, no. 5, pp. 332-340, 1998.

[11] D. T.-Y. Chiu and T. Z. Liu, "Free radical and oxidative damage in human blood cells," Journal of Biomedical Science, vol. 4, no. 5, pp. 256-259, 1997.

[12] H. M. Schipper and S. Cisse, "Mitochondrial constituents of corpora amylacea and autofluorescent astrocytic inclusions in senescent human brain," GLIA, vol. 14, no. 1, pp. 55-64, 1995.

[13] S. Scheibe, M. M. Dorostkar, C. Seebacher, R. Uhl, F. Lison, and J. Herms, "4D in in vivo 2-photon laser scanning fluorescence microscopy with sample motion in 6 degrees of freedom," Journal of Neuroscience Methods, vol. 200, no. 1, pp. 47-53, 2011.

[14] X. Li, Z. Xue, and S. Zhang, "Construction of recombinant plasmid harboring APP717 mutation and preliminary study of APP proteolysis," Journal of Huazhong University of Science and Technology: Medical Science, vol. 27, no. 5, pp. 487-490, 2007.

[15] R. P. Brendza, K. Simmons, K. R. Bales, S. M. Paul, M. P. Goldberg, and D. M. Holtzman, "Use of YFP to study amyloid- $\beta$ associated neurite alterations in live brain slices," Neurobiology of Aging, vol. 24, no. 8, pp. 1071-1077, 2003.
[16] C. Meng, Z. He, and D. Xing, "Low-level laser therapy rescues dendrite atrophy via upregulating BDNF expression: implications for Alzheimer's disease," Journal of Neuroscience, vol. 33, no. 33, pp. 13505-13517, 2013.

[17] X. Yang, S. Askarova, W. Sheng et al., "Low energy laser light $(632.8 \mathrm{~nm})$ suppresses amyloid- $\beta$ peptide-induced oxidative and inflammatory responses in astrocytes," Neuroscience, vol. 171, no. 3, pp. 859-868, 2010.

[18] J. Liang, L. Liu, and D. Xing, "Photobiomodulation by lowpower laser irradiation attenuates $\mathrm{A} \beta$-induced cell apoptosis through the Akt/GSK $3 \beta / \beta$-catenin pathway," Free Radical Biology and Medicine, vol. 53, no. 7, pp. 1459-1467, 2012.

[19] M. A. Bellgrove, J. G. Phillips, J. L. Bradshaw, K. A. Hall, I. Presnell, and H. Hecht, "Response programming in dementia of the Alzheimer type: a kinematic analysis," Neuropsychologia, vol. 35, no. 3, pp. 229-240, 1997.

[20] D. Litscher and G. Litscher, "Laser therapy and strokequantification of methodological requirements in consideration of yellow laser," International Journal of Photoenergy, vol. 2013, Article ID 575798, 4 pages, 2013.

[21] S. E. Harris, J. E. Field, and A. Imamoğlu, "Nonlinear optical processes using electromagnetically induced transparency," Physical Review Letters, vol. 64, no. 10, pp. 1107-1110, 1990.

[22] S. Weis, R. Rivière, S. Deléglise et al., "Optomechanically induced transparency," Science, vol. 330, no. 6010, pp. 1520-1523, 2010.

[23] M. Scherman, O. S. Mishina, P. Lombardi, E. Giacobino, and J. Laurat, "Enhancing electromagnetically-induced transparency in a multilevel broadened medium," Optics Express, vol. 20, no. 4, pp. 4346-4351, 2012. 

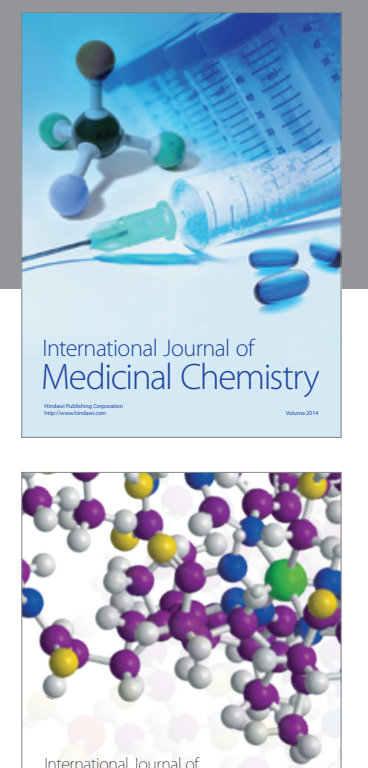

\section{Carbohydrate} Chemistry

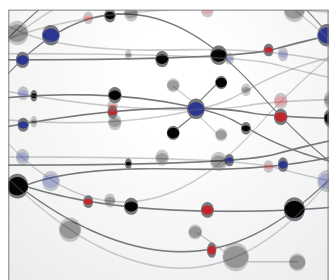

The Scientific World Journal
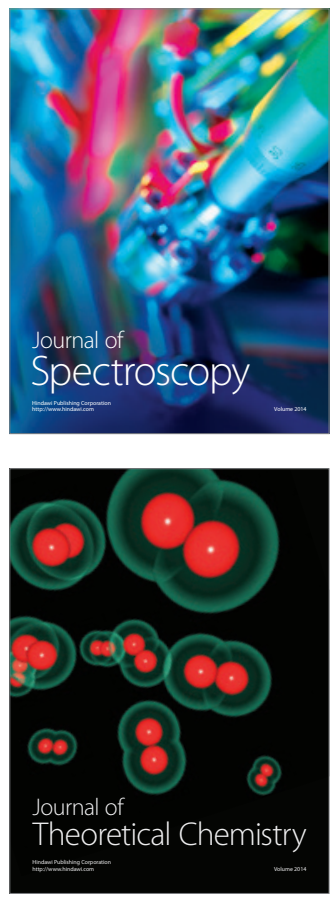
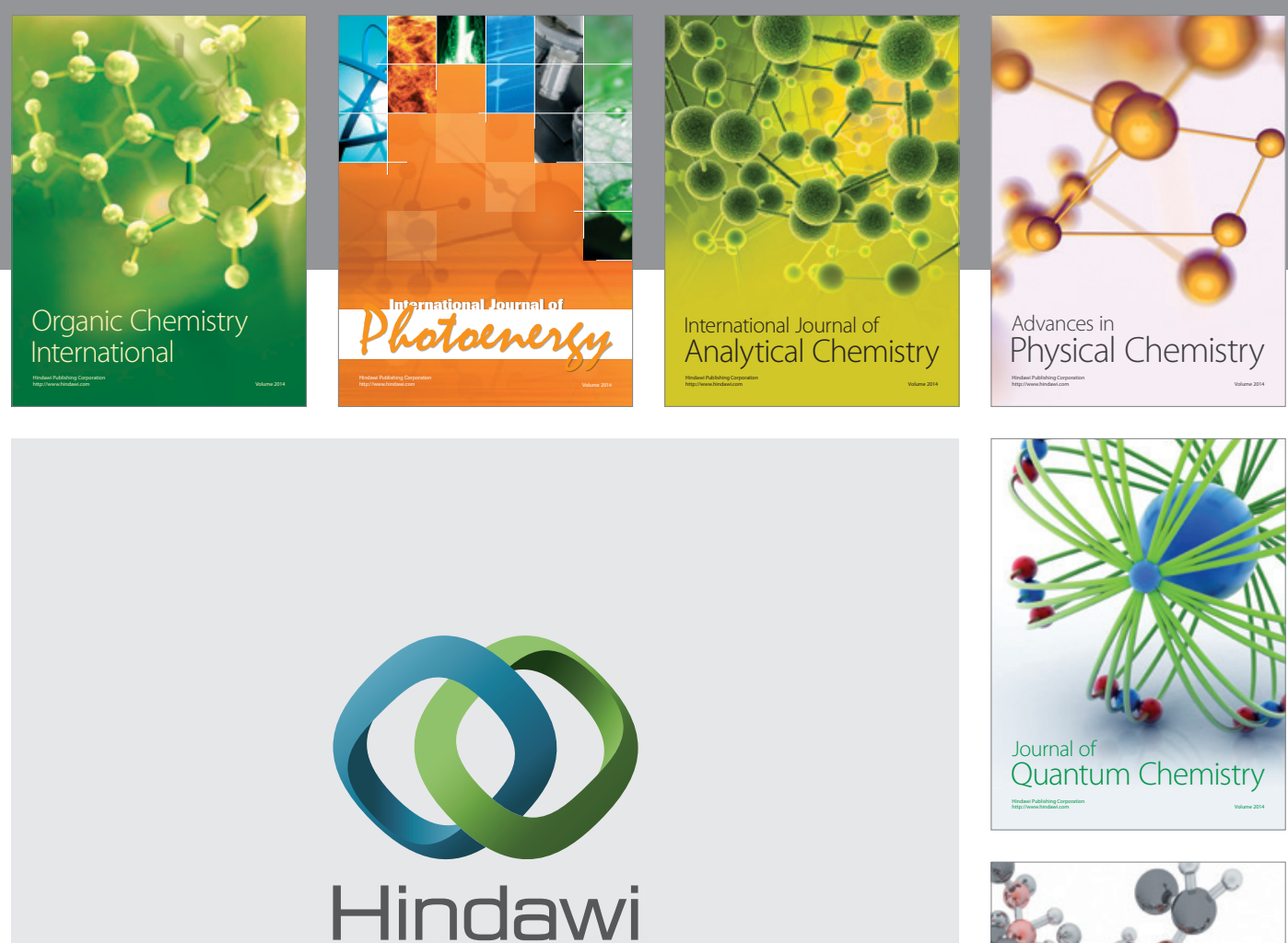

Submit your manuscripts at

http://www.hindawi.com

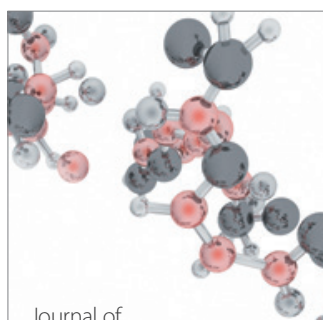

Analytical Methods

in Chemistry

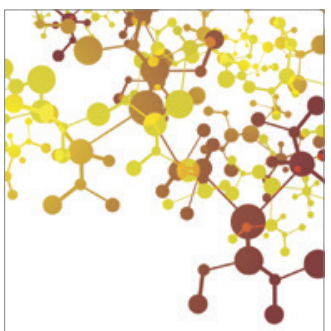

Journal of

Applied Chemistry

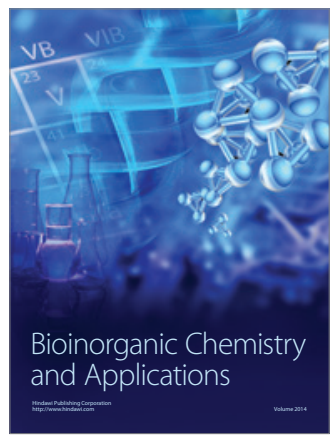

Inorganic Chemistry
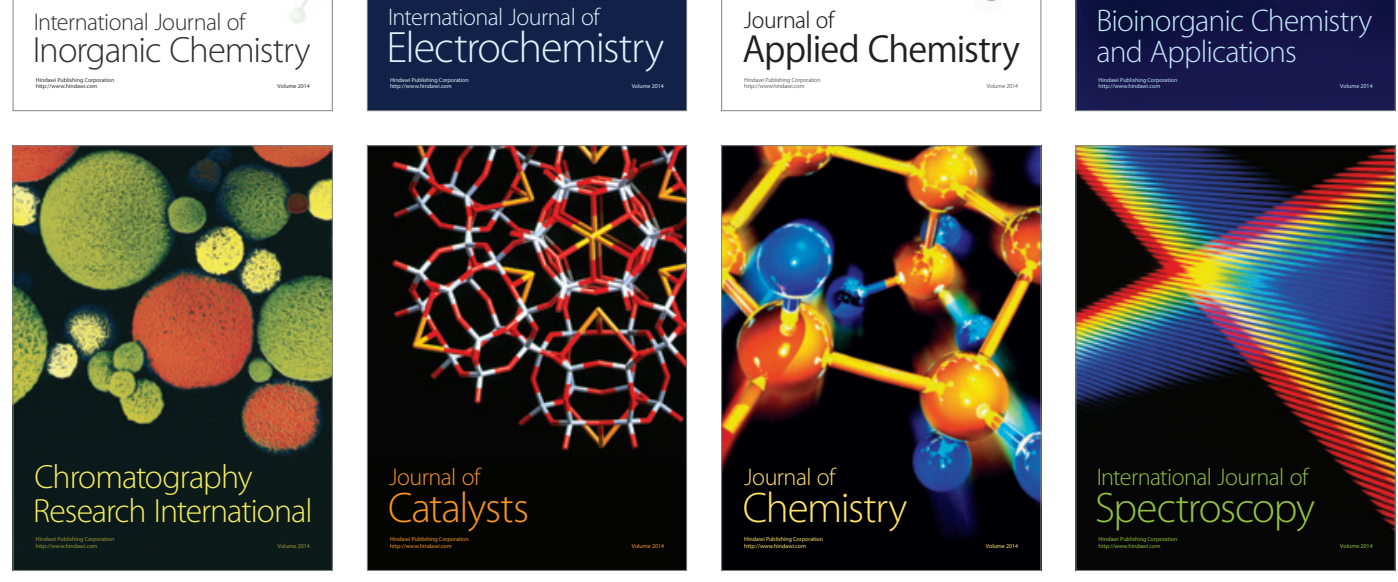